\title{
Investigation to isolate the acute effects of carbohydrate restriction in energy matched diets on postprandial substrate metabolism: preliminary data from a cross-over dietary intervention
}

\author{
H. Biyikoglu, A. Collins and M.D. Robertson \\ Faculty of Health and Medical Sciences, University of Surrey, Guildford, Surrey, UK
}

Intermittent energy restriction (IER) diets have been found to be successful in improving metabolic disease markers ${ }^{(1)}$ and affect postprandial glucose-lipid metabolism in the short term ${ }^{(2)}$. Given that carbohydrate plays a key role in glucose homeostasis and substrate handling, and the restricted periods of IER are inherently low in carbohydrates, it is of interest to determine whether the metabolic alterations of energy restriction are elicited simply due to the reduced availability of carbohydrate.

Therefore, this study aimed to investigate the short-term effects of carbohydrate restriction in comparison to an isocaloric energy balanced diet.

Five (one male) healthy $(27.2 \pm 9.68 \mathrm{y} ; 24.88 \pm 1.96 \mathrm{~kg} / \mathrm{m} 2)$ subjects were recruited into this acute, two- way cross-over study. Subjects consumed two pre-prepared intervention diets for one day (36hr) separated by a 5-days washout period: normal carbohydrate ( $55 \%$ of energy) energy balanced diet (nEB), and low-carbohydrate (50g/day) energy balanced diet (LCEB). Glucose levels were measured during a 3-day trial period via fitted continuous glucose monitors (CGM). Following both 36 hours of LCEB and nEB blood metabolites were measured in the morning fasted state and serially across 360 minutes postprandially. At these same points, substrate utilisation (RQ) and energy expenditure were assessed by indirect calorimetry. Appetite responses were examined with ad libitum lunch on the study day and over a 3-day period. Data were analysed using repeated-measures ANOVA and Wilcoxon signed-ranks and presented as mean \pm SEM.

CGM measurement during the intervention day showed significantly lower glucose levels during the LCEB leg $(p<0.01)$. Following a 36-hours of a low-carbohydrate (LCEB) diet had lowered postprandial triacylglycerol by $20 \%(p=0.006)$. However, fasting levels of triacylglycerol were similar between the two diets $(p=0.063)$. Similarly, there was no statistical effect on fasting glucose levels $(p>0.05)$ and postprandial glucose response to the test meal $(p=0.064)$. Presumably, the sample size may have been too small for the difference in fasting triacylglycerol and postprandial glucose levels between legs to reach statistical significance. As anticipated, hepatic production of postprandial $3-\beta$-hydroxybutyrate $(3-\beta O H B)$ was elevated in the LCEB leg $(\mathrm{P}=0.043)$, but fasting results showed no effect of diet on $3-\beta O H B(p>0.05)$. No significant differences in resting energy expenditure or fasted RQ were observed between legs, although carbohydrate restriction significantly lowered postprandial RQ $(P=0.040)$.

This preliminary data suggests that carbohydrate restriction without energy restriction may mimic the acute metabolic response observed in intermittent energy restriction. Further studies are planned to improve the sample size and compare the acute effects of carbohydrate restriction with and without energy restriction.

\section{References}

1. He SS, Wang JA, Zhang J et al. (2021) Obesity 29,108-115.

2. Antoni R, Johnston KL, Collins AL et al. (2016) Br J Nutr 115, 951-959. 